\title{
Chemical Hazards of Nanoparticles to Human and Environment (A Review)
}

\author{
FOZIA HAQUE KHAN \\ Optical Nanomaterials Laboratory, Department of Physics, \\ Maulana Azad National. Institute of Technolog, Bhopal - 462 051, India. \\ ${ }^{*}$ Corresponding author: foziazia @ rediffmail.com \\ http://dx.doi.org/10.13005/ojc/290415
}

(Received: September 25, 2013; Accepted: November 25, 2013)

\begin{abstract}
Science and technology have identified unique properties of nanomaterials. These properties may yield many far-reaching societal benefits, but they can also pose hazards and risks. Although the nanotechnology industry is still in its infancy, as more nanotechnology applications are commercialized, the potential for human exposure to nanoparticles and raw nanomaterials would continue to increase. One area of most concern about hazards is the workplace-be it a research laboratory, start-up company, production facility, or operation in which engineered nanomaterials are processed, used, disposed, or recycled. In order to determine whether the unique chemical and physical properties of new nanoparticles result in specific toxicologic properties, the nanotechnology community needs new ways of evaluating hazards and ultimately assessing the risk factor and therefore an attempt must be made to exclusively concentrate on the potential health hazards of nanopowders. One potential hazard that appears to have received little attention to date is their explosibility. This literature review has been commissioned to explore the physical and chemical properties of nanoparticles that could differentially influence toxicity, use of nanoparticles in industry and the potential hazards.
\end{abstract}

Key words: Nanoparticles; Hazards; Toxic \& explosive nanoparticles; Drug delivery.

\section{INTRODUCTION}

A nanometre $(\mathrm{nm})$ is defined as the millionth part of a millimetre or 10-9 $\mathrm{m}$. In comparison, the diameter of a hair is approximately $50,000 \mathrm{~nm}$; and that of a hydrogen atom approximately $0.1 \mathrm{~nm}$. Fundamental for nanotechnology is the fact that new functionalities or properties result from the nanoscale character of the components. New properties and/or functionalities can be related to scratch resistance, colourfulness, transparency, conductivity, strength etc. These new functionalities result for the most part from the altered ratio of surface atoms to volume atoms and from the quantum-mechanical behaviour. Nanoparticles are understood to be both natural and artificially manufactured particles smaller than 100 $\mathrm{nm}$.

Man has been using nanopowders, in the form of nano-sized pigment and carbon colourants since the beginning of recorded history. It is only, however, since the early twentieth century that nanopowders have been manufactured in bulk, for example carbon black (produced by the burning 
of natural gas) for use as a reinforcing agent in vehicle tyres. The real growth in the production of nanopowders, though, did not start until the mid1970 's. The current use of nanopowders is extremely broad and there are very few commercial products that do not include nanoparticles. Quantities of nanopowders produced can range from large quantities (tons) of products like fumed silica and carbon black [Warheit 2004 a,b] to extremely small quantities of the more precious products, for example stabilised metallic quantum dots and atomic clusters.

Moreover, nanotechnology is distinguished by innovative manufacturing options it offers. These include, in particular, improved control possibilities in the manufacturing process and self-organisation of nanoscale materials. In self-organisation, under certain ambient conditions, nanoscale materials join to form new structures [Warheit, et al., 2004]. The particular potential of nanotechnology lies in the combination of a topdown approach, with a bottomup approach. In Topdown method, the required nano structures are obtained by means of progressive miniaturization, while bottomup technique supports the scheme of combining individual components. Nanoparticles occur naturally too, for example minute particles of salt become airborne through the action of the waves of the sea, or are an unintended but largely unavoidable by-product of combustion and other high-temperature industrial processes [Beck H. et al., 1997].

Along with the increasing production and use of nanoscale particles there has been a growing concern over the impact of this new technology on health \& safety and the environment [Steinfeld et. al., 2004, Swiss Re 2004]. This has almost exclusively concentrated on the potential health hazards of nanopowders [Daar A et al 2003, Ameen et al., (2013) and Zhoq et al., (2012)]. For assessing the sustainability effects through the production and application of nanotechnological products, a life-cycle analysis of products already in use, should be carried out by setting prospective positive ecological effects against potentially problematic effects. At the same time it should be kept in mind that the assessment would depend on the known material and energy data and does not allow any estimate to be made concerning products still under development.

\section{Nanopartilces and Properties}

Nanoparticles are understood to be both natural and artificially manufactured particles smaller than $100 \mathrm{~nm}$. By synthetic nanoparticles, we mean artificially manufactured particles smaller than $100 \mathrm{~nm}$ in size, which have changed properties and/or functionalities. They are referred to as engineered nanoparticles or nanosized particles. The terms "ultrafine particles" (especially used by toxicologists), "nucleation mode particles" or "engineered nanostructured material" are also used (Oberdörster et. al., 2005)

The properties already known with respect to atoms or molecules, such as electrical conductivity, magnetism, colour, mechanical hardness or a particular melting point are changed depending on the size or structure of the particles. Cadmium telluride particles (CdTe), for example, fluoresce in different colours depending on the size of the particles (2 to $5 \mathrm{~nm}$ ) [Tang et al., 2002]. Three basic property changes are observed:

1. a changed quantum-mechanical behaviour, e.g. in relation to colour, transparency, hardness, magnetism and electrical conductivity

2. an enlarged surface leading to changes in the melting and boiling point, the chemical reactivity and the catalytic effect and

3. a changed molecular recognition capability, which, combined with an increased recognition ability, adaptation ability, repair ability and selforganisation, opens up new biological applications.

Materials currently being produced as nanopowders include: metals; metal oxides, borides, carbides, nitrides and sulphides; and, non-metal materials such as various forms of carbon, clay silica and silicon carbides. Most nanopowders are usually produced in spherical form, but nanosized particles can also be produced as flakes (eg aluminium), fibres (eg Teflon and other polymers) or tubes (eg carbon nanotubes). In the case of fibres and tubes the lengths can fall well outside the nanoscale. For example carbon nanotubes can be produced with diameters down to $1 \mathrm{~nm}$, though the lengths can be up to $1 \mathrm{~mm}$. The literature search found little information on the quantities of nanopowders that are produced or used by industry. What is known is that the production and use of the nanopowders that have an established use, eg materials like 
carbon black, silica, titanium oxide and zinc oxide, is in tonne quantities. While at the other end of the scale for some of the more exotic materials that are now being produced as nanopowders, the amounts being produced and used are no more than gramme quantities.

A classification of nanomaterials under the categories of dimension, phase structure and manufacturing process.

- Nanosystems can be nanoscale structured in one or several dimensions:

- One-dimensional nanostructured systems, e.g. nanowires, quantum wires

- Two-dimensional nanostructured systems, e.g. ultrathin films

- Three-dimensional nanostructured systems, e.g. quantum points nanopowder, functional supramolecural systems.

- $\quad$ They can consist of one (single-phase solids) or several (multi-phase solids) solid phases or of different phases (multi-phase systems, solid-liquid, liquid-gaseous, solid-gaseous).

In addition, classification can be made according to the manufacturing process (mechanical or in gaseous or liquid phases). Hansen and his colleagues [? ref]suggest that nanomaterials can be categorized depending on the location of the nanoscale structure in the system. This leads to a division of nanomaterials into three main categories, which then can be further divided into subcategories:

- Materials that are nanostructured in the bulk;

- Materials that have nanostructure on the surface; and

- Materials that contain nanostructured particles.

Many of these materials if finely divided with particle sizes in the micron-range and dispersed in air, given the right conditions, can form explosive clouds.

\section{Applications}

Nanopowders are already used in a wide range of industries and developments in the use of nanomaterials are being reported almost on a daily basis in the scientific literature. Current applications include:

- $\quad$ Sunscreens and cosmetics. Titanium and zinc oxide nanopowders are widely used in sunscreens and other creams and lotions. Textiles. Teflon and other polymer nanoscale fibres are used in some stain and wrinkleresistant clothing.

Coatings. Custom-made nanocomposites and titanium dioxide nanopowders are used in anti-scratch coatings for lenses and to treat glass surfaces to give them self-cleaning properties. New anti-graffiti coatings for walls contain nanoparticles.

Sports goods. Carbon nanotubes and nanoclay particles are used in the manufacture of tennis racquets and tennis balls. Ski waxes based on nanocomposites are also available.

Explosives, propellants and pyrotechnics. Aluminium nanopowders are being used instead of micron-scale powders to further enhance the burning characteristics of these materials.

Catalysts. Their performance is being enhanced by using catalysts composed of nanosized materials - the greater the surface area the more effective a catalyst will be.

Electronic storage media. Hard-disks based on nanostructured magnetic multi-layers currently dominate the market.

Filtration. Filters made of nanofibres, for example alumina fibres, are used to trap materials such as viruses, bacteria, animal cells, endotoxins and macromolecules such as DNA. They are also used for removing trace toxic metals from water.

Disinfectants. Nanoemulsions and nanoparticles of a lanthanum-based compound are being used in surface disinfectants and to prevent the growth of algae in pools and aquariums.

Medical uses. Silver nanoparticles are used as an antibacterial agent for bandages and in the coatings on hearing aids.

Alloys and metals. Alloys and metals are being produced with nano-crystalline structures to increase their strength. For example nanocrystalline nickel is as strong as hardened steel and nanocrystalline aluminium alloys can be up to twice as strong as their conventional equivalents. 
- $\quad$ Non-metallic components. Carbon nanotubes are being used to increase the strength of automobile plastic components. Nanoparticles of diamond are being added as reinforcing agents to rubbers, plastics and resins.

- Abrasives. Abrasive pastes made from diamond nanopowders are being used for high precision polishing of lenses and mirrors in optical instruments.

Lubricants. The lubricating properties of oils are being improved by the addition of diamond nanopowders.

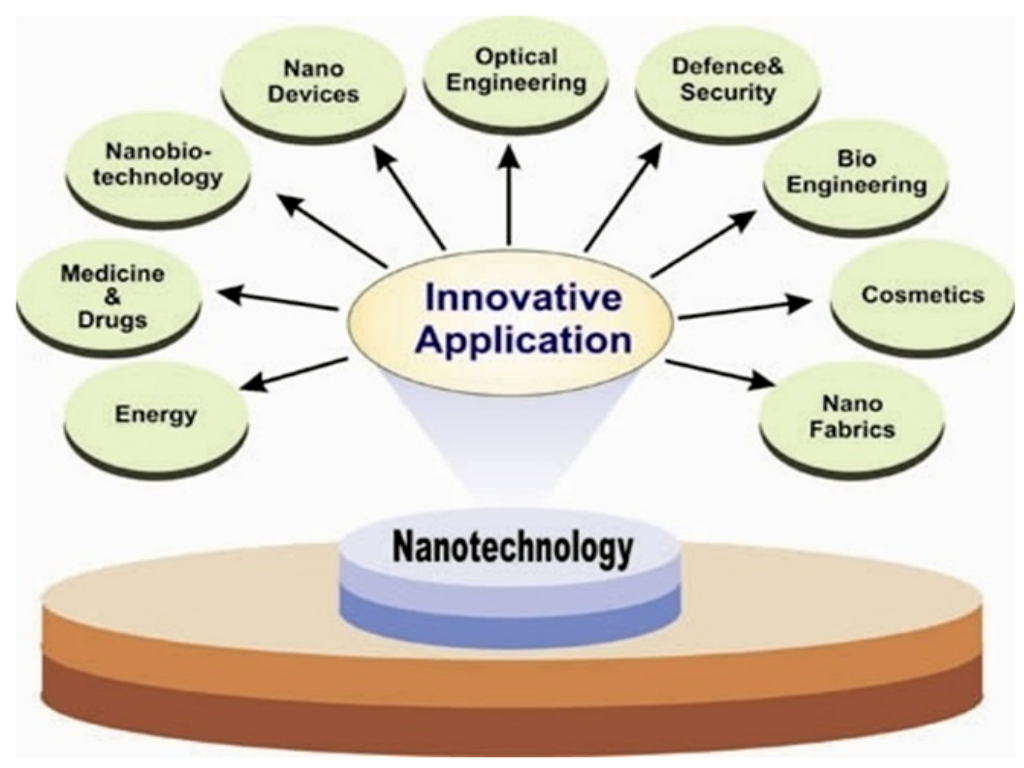

Fig. 1: Various applications of nanotechnology

Applications currently under development, some of which are likely to be commercially available in the next year or so, include:

- Batteries. Prototype batteries using nanoparticles have been developed that offer ten times the charge rate of conventional rechargeable batteries.

- $\quad$ Solar cells. Cells based on tiny structures called nanorods, that can be tuned to respond to different wavelengths, are currently under development. Another type of cell under development is one that produces hydrogen rather than electricity.

- $\quad$ Fuel cells. Nanostructured catalysts look as if they may be a critical component in finally making fuel cells a reality. The other impact nanotechnology will have is on the way fuel is stored in the fuel cells. The nanotube shows promise as a storage medium for hydrogen and there are plans to shortly launch a methane-based fuel cell for powering mobile phones and laptops, which will use nanohorns, a relative of nanotubes, for the fuel storage.

Light sources. A light bulb, driven by nanotubes, which promises to match or exceed the efficiencies achieved with fluorescent lighting is under development.

Electronic storage media. A number of nanotechnologies are promising new types of RAM for computers that would be non-volatile (ie the information stored in the RAM would not be lost when the power is turned off) and could have enough capacity to make disc storage unnecessary for applications such as personal computers. One example of this type is magnetic RAM (MRAM), which has already seen some limited commercial use.

Display technologies. Flat-panel electronic displays, for example a hang on the wall television screen, based on nanotubes, will soon be commercially available. 
- $\quad$ Bioanalysis and biodetectors. Nanoparticles small enough to behave as quantum dots can be made to emit light at varying frequencies. Efforts to exploit this property for bioanalysis are being pursued by a number of companies. For example, by getting particles that emit at different frequencies to attach to different molecules it would then be possible to spectroscopically determine the presence of many substances in one sample. Others are exploiting the sensitivity of the electrical properties of nanowires and nanotubes to develop highly sensitive biodetectors that could reveal the presence of a single molecule. Nanostructured materials, coupled with liquid crystals and chemical receptors offer the possibility of cheap, portable biodetectors that might, for instance, be worn as a badge.

- $\quad$ Drug delivery. Drug delivery is an area that is already showing significant impact from nanotechnology, with some approaches using nanoparticles or nanocapsules to deliver drugs through the skin, lungs, stomach and eyes already in clinical trials and many more in pre-clinical trials. The potential advantages of these approaches are many and include increased solubility and resistance to gastric enzymes (offering oral delivery of drugs that previously needed intravenous delivery), controlled release and the ability to direct the drug, by various means, to where it is needed.

- Medical implants and new organs. In the longer term there is the potential to produce longer lasting medical implants and to even grow new organs using nanotechnology. This will result from the ability to produce stronger structures and to produce surface properties designed to encourage or discourage cell growth or increase biocompatibility.

The above list of applications is by no means exhaustive, but it shows how broad is the current use of nomaterials and some of the potential new areas of application and for making important risk plan too.

Lack of information: particle properties, characterization and metrology

There is a lack of reliable, affordable and standardized measurement methods for (a) measuring nanoparticle size and shape; and (b) characterizing nanoparticles, e.g. their composition and surface behavior. Addressing these gaps are essential for conducting meaningful and valid research that produces results that are comparable, repeatable and accepted by the scientific community, and on which a reliable system of risk identification, assessment and management can be based. Associated with this is the need to define the most appropriate particle metric or metrics for use in exposure and hazard studies. It is important to be clear about what is going to be measured, why a particular metric has been chosen and how to carry out measurements. It is possible that the most suitable metrics will differ for different types of nanoparticles; for example, surface area for nanoparticles and fibre number for nanotubes. There is at the same time a need to establish standardised, wellcharacterised nanoparticles that vary in size, shape, durability, composition and surface reactivity for use in metrology, characterisation, exposure and hazard assessment notably for comparative benchmarking purposes in toxicology.

\section{Exposure}

In taking an approach for assessing and managing the risk based impact of nanoparticles, it is important to understand the nature and extent of human and environmental exposure. This provides information on where any potential risk is most likely to occur [Mark et al., 2005]. These are:

- occupational exposure in the workplace (human);

exposure from deliberate environmental releases, e.g. remediation of contaminated groundwaters and land (environment and possibly human);

exposure from 'unintentional' environmental releases, e.g. from fuel additives and in industrial and domestic waste streams (environment and human); exposure from consumer products, such as cosmetics (human); and exposure from medical products, including drugs, treatments and devices (human). atmosphere is a major route of human and environmental exposure to particulates, in particular through inhalation [Oberdörster 2000]. 


\section{Production techniques}

The key new factor distinguishing nanotechnology is the controllable manipulation of atoms and molecules on the nanoscale This will increasingly enable the construction of functional nanostructures atom by atom and consequently the creation of materials and components with hitherto unknown properties. There are two basic approaches to the production of nanomaterials. The first is the 'top-down' approach, which involves the breaking down of the bulk material into nanosized structures or particles. These techniques are an extension of those that have been used for producing micronsized particles. An example of such a technique is high-energy wet ball milling. The alternative approach, which has the potential of creating less waste and hence is more economical, is the 'bottom-up' approach. In this approach individual atoms or molecules are built up to form the require nanostructure or nanoparticles. Many of these techniques are still under development or are just beginning to be used for commercial production of nanopowders. Bottom-up approaches, in current use or at an advanced stage of development, can be classified into liquid, vapour or solid phase techniques.

- $\quad$ Liquid phase techniques: Wet chemistry techniques that have been used for producing larger particles are being adapted to produce nanosized material, the product crystallising or precipitating out of the solution. In order to achieve the reduction in particle size the reaction chemistry must be chosen to provide a fast spontaneous reaction and also be able to limit subsequent growth of the particles after nucleation. The particle size can be controlled by the use of various polymers, gels or microemulsions to constrain the growth of the particles. An example of a liquid phase technique is the Sol-Gel process[ hs4]. This process is used for making ceramic nanomaterials and involves converting from a liquid 'sol' phase (mainly colloidal) to a solid 'gel' phase.

- Vapour phase techniques:There are a number of techniques that can be classed under this category, but they all involve two basic steps. The first step is the vaporisation of the material followed by a rapid controlled condensation to produce the required size of particle. In some techniques the powder formed is the same composition as the starting material, while others rely on decomposition occurring during the vaporisation step to produce the desired product. Production of carbon black, obtained by collecting the soot produced by the burning of natural gas or other hydrocarbons, is probably the earliest example of a vapour phase technique. Silica (silicon dioxide) and titanium dioxide are also produced in bulk by a combustion (flame synthesis) process.

Solid phase techniques: An example of this technique is mechanochemical processing (MCP)12 that is being used to produce metal oxide nanopowders. Dry milling is used to induce chemical reactions through ballpowder collisions that result in nanoparticles being formed within a salt matrix. The particle size produced depends on the chemistry of the reactant mixture, milling and heat treatment conditions. Particle agglomeration is minimised by the salt matrix, which is then removed by a simple washing procedure.

\section{Hazards to humans and the environment}

Present article reports and identifies, as a research priority, the need to understand the hazards of nanoparticles that can pose to human health as a result of consumer, medical, occupational and environmental exposure.

\section{Hazards to human}

- Toxicokinetics - entry into and distribution around the human body: A primary hazard scoping study is to further research into the so called 'toxicokinetics' of nanoparticles associated with exposure via the lung, skin and gut (see Figure 1). In essence, toxicokinetics look at how a particle may get into the body, how it is circulated and distributed within it, and how it may be metabolised and excreted. Understanding this is important as it allows consideration of the important target organs that may or may not be affected, to predict realistic exposure doses and to understand how the body responds to nanoparticle exposure in terms of metabolism and excretion. To date, few, if any, adsorption, distribution, metabolism and excretion (ADME) studies have been 
conducted for nanoparticles [Kreuter, Jörg et. al.,].

- Movement within and between cells and their cellular toxicity: The scoping study emphasised the need to understand the localisation of nanoparticles within cells and their cellular toxicity. In particular, transport of nanoparticles across membranes both between and within cells (e.g. into mitochondria) and an understanding of their toxic effects (e.g. oxidative stress, genotoxicity, inflammatory cytokine production, apoptosis) are important. Such in vitro cell studies provide an understanding of the mechanisms of toxicity, how cellular defence mechanisms respond and possible pathogenic effects. This will lead to the selection of relevant endpoints for standard methods to assess hazard (see below). Within this, the role of particle size, composition, aggregation and disaggregation should be considered and the nature and magnitude of biochemical responses that influence cellular fate, biopersistence and degradation studied [Lam, Chiu-Wing et. al.,].

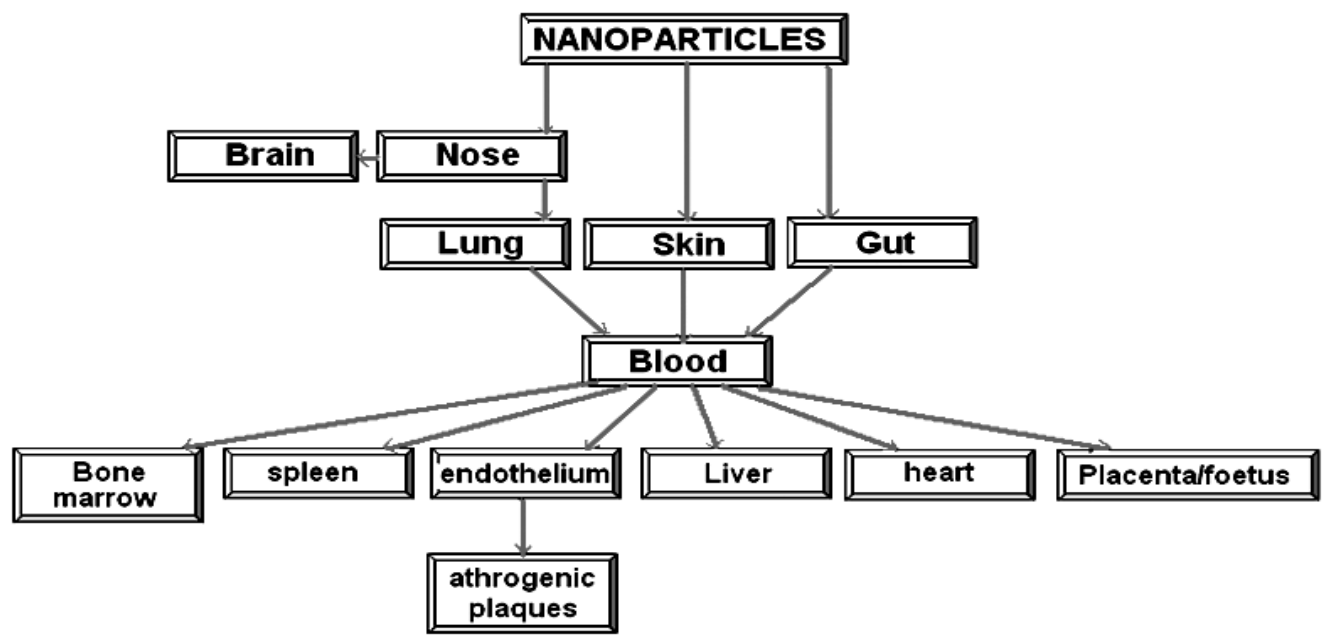

Fig. 2: Summary of the hypothetical toxicokinetic pathways for nanoparticels

- Respiratory system, cardiovascular system and brain: Given our understanding of the relative importance of potential exposure in the workplace and in the environment via the air, a particular focus of research should be in the airways and lung. Here there is good evidence of pathogenic effects from non-engineered nanoparticles. Most of the available information concerns the effect of ultrafine combustion products on inhalation toxicology. Some data, partly inferred from particulate pollution studies and supported by work on combustion derived carbon nanoparticles, indicate that these can have inflammatory effects in the lung. This inflammatory response has been observed to be greater for nanoparticles than for the same mass of larger particles of that substance, but has not been observed for all particle types. This supports the suggestion that particle chemistry and surface characteristics as well as size and dose determine toxicity. Other work has also shown that some nanoparticles can pass to the brain via the central nervous system following inhalation; while the exact pathway for this is uncertain, the olfactory nerve is considered as one mechanism.

Skin, gut and other organs: As discussed above, current uses of nanoparticles in consumer products and concerns regarding occupational exposure point to the skin as being an important portal of entry for the human body. However, the extent to which nanoparticles are able to penetrate the skin and cause adverse effects is not fully understood. For the gut, there is good 
evidence that larger microparticles cross the gut barrier under normal conditions, but no evidence exists as to whether this is increased in the case of nanoparticles. There is insufficient evidence to determine whether nanoparticles adversely affect the gut [Derfus, A.M. et. al].

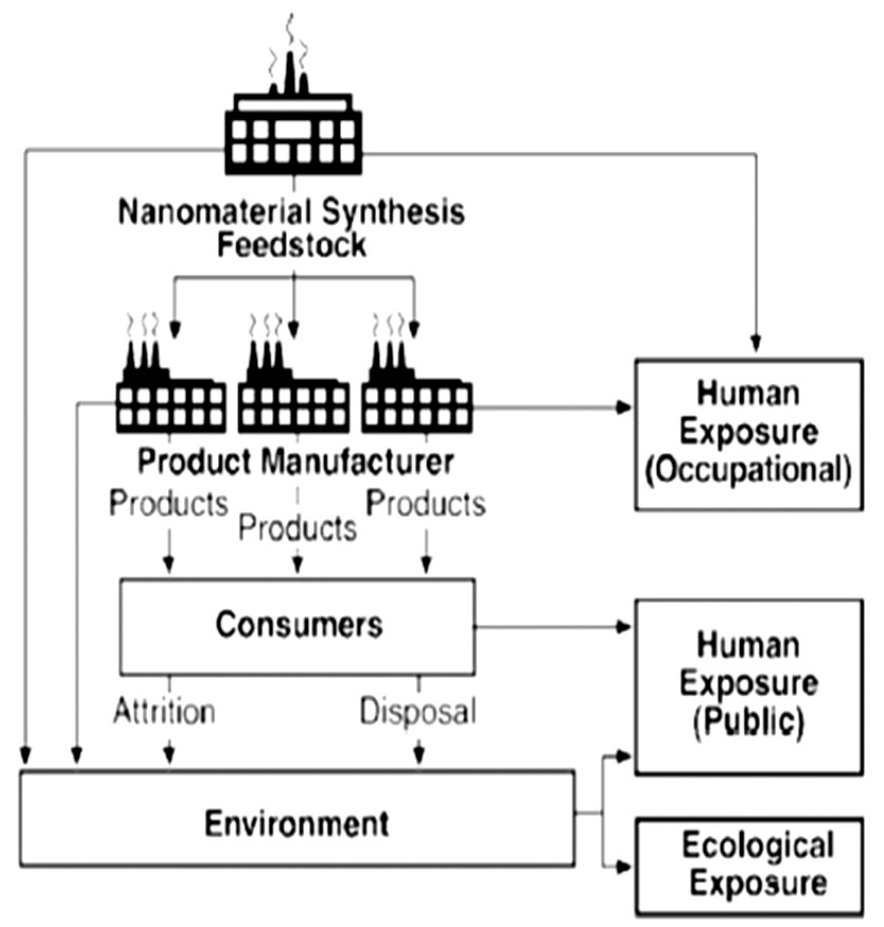

Fig. 3: Potentials to release and exposures

\section{Environmental hazards}

The natural environment is composed of many complex ecosystems comprising atmospheric, terrestrial, fresh water and marine compartments. Unlike human exposure, the number of species potentially at risk from nanoparticles is extremely large. Hazards associated with nanoparticle exposure can potentially act at individual or population level and might also impact on the structure and function of the ecosystem as a whole. Until a comprehensive understanding of sources of nanoparticles to the environment and a complete understanding of their basic environmental fate and behaviour is understood, it is hard to identify which, if any, components of these ecosystems (soils, surface waters, air) are at risk of contamination from nanoparticles [Glor M. 1988].

- Effects in groundwaters and soils: A research priority should be to understand the environmental fate and behaviour of such nanoparticles in soils and groundwaters.
The uptake and toxicity of nanoparticles to soil flora and fauna should also be considered. The environmental fate and toxicity of nanoparticles to microbes within sewage treatment works can also need to be considered, particularly once sources of these to sewer have been confirmed [Chen $\mathrm{HH}$ et al].

Ignition and explosion potential: One specific, potential hazard posed by nanoparticles for which a detailed understanding of their properties is urgently needed, is the potential to cause fire or explosion. A similar effect is observed in larger particles when they are released to the atmosphere and subject to an ignition source. A study undertaken by the HSE (Health and Safety Executive) indicated that nanoparticles are almost certain to give rise to a dust explosion hazard and that due to their large specific surface area they may well be spontaneously flammable on exposure 
to air. This is particularly the case with metal nanoparticles as they oxidise easily [Eckhoff $\mathrm{R} F$ et al 2004].

- Wider effects associated with unintentional release: There is poor understanding of the uptake and toxicokinetics of nanoparticles in microorganisms, invertebrates, vertebrates and plants. At the cellular level there is also poor understanding of cellular uptake, localisation and toxicity. The effects at low doses and over longer timescales should be considered. Exploring the combined effects of nanomaterials and other contaminants, such as metals and organics, should also be considered. There is also the potential to use mammalian toxicology data to inform ecotoxicology. Toxicological information from the effects of particle chemistry and physical characteristics, cellular effects and toxicological mechanisms may impact at cellular and molecular levels with common cellular responses.

An understanding of particle physicochemical properties and basic mechanisms of toxicity can lead to the definition of appropriate endpoints for standard tests used in hazard assessment.

\section{CONCLUSIONS}

This article has summarised our current state of knowledge on the potential risks posed by nanoparticles. The information will make an important contribution to the evidence base on which we can develop policy to ensure the responsible management and control of the potential risks posed by nanoparticles.

A key part of this will be giving the information on the social and ethical implications of nanotechnologies. Our understanding is perhaps most advanced in the area of human toxicology, and in particular, the effects resulting from the inhalation of nonengineered nanoparticles. Here, as with the other areas covered in the article, much of our knowledge is derived from studies of combustion product particles in urban environments.

A priority, in the first instance, is to identify suitable metrics and develop the methods with which to measure, characterise and assess the behaviour and properties of nanoparticles. Establishing freely available, standard reference materials for research purposes will be an important component of this.

\section{REFERENCES}

1. Beck $\mathrm{H}$, Glienke $\mathrm{N}$ and MohImann $\mathrm{C}$. Combustion and explosion characteristics of dusts. BIA-Report 13/97, HVBG, November (1997).

2. Chen $\mathrm{HH}$ et al. Acute and subacute toxicity Study of water-soluble polyalkylsulfonated C60 in Rats. In: Toxicol Pathol. 26(1): 143151(1998)

3. Daar A, Mnyusiwalla $A$ and Singer $P$. Chemistry in Britain Editorial, (2003).

4. Derfus, A.M. et. al. Probing the cytotoxicity of Seminconductor Quantum Dots. In: Nano Lett. 4(1): 11 (2004)

5. Eckhoff R F. Dust Explosions in the Process industries (3rd Edition), Gulf Professional Publishing, ISBN 0-7506-7602-7 (2003).

6. Glor M. Electrostatic hazards in powder handling. Research Studies press Ltd, John Wiley \& Sons Inc., (1988).
7. Hansen and his colleagues???

8. HSE, Report Explosion hazards associated with nanopowders (EC/04/03). London: HSE (2005).

9. Kreuter, Jörg et. al. Direct evidence that polysorbate-80-coated poly (butylcyano acylate) nanoparticles deliver drug to the CNS via specific mechanisms requiring prior binding of drugs to the nanoparticles. Pharm Res 20(3) pp. 409-416 (2003).

10. Lam, Chiu-Wing et. al. Pulmonary Toxicity of Single-Wall Carbon Nanotubes in Mice 7 an 90 Days after Intratracheal Instillation. Toxicol. Sci. 77(1): 126 (2004)

11. Sadia Ameen and Hyungshikshin., Orient. J. Chem., 29(3): 837-860 (2013).

12. G. Zhou and W. Wang., Orient. J. Chem., 28(2): 651-655 (2012).

13. Mark, D., et al., A scoping study to identify 
exposure data needs for addressing the risks presented by nanoparticles and nanotubes. Buxton: Health and Safety Laboratory (2005).

14. Oberdörster, Günther, Pulmonary effects of inhalated ultrafine particles. In: Int Arch Occup Environm Health. 74(1) pp. 1-8 (2000).

15. Oberdörster, Günther; Oberdörster, Eva; Oberdörster, Jan Nanotoxicology: An Emerging Discipline Evolving from Studies of Ultrafine Particles. In: Environmental Health Perspectives. 113(7): 823-839 (2005).

16. Schulte, Paul A. ; Salamanca-Buentello, Fabio, Environ Health Perspect., 115: 5-12 (2007).

17. Steinfeld, Michael; von Gleich, Armin; Petschow, Ulrich; Haum, Rüdiger; Chudoba,

18. Swiss $\mathrm{Re}$ [Ed.] Nanotechnologie: Kleine Teilchen - große Zukunft? Reihe Risk
Perception Zürich (2004) .

19. Thomas; Haubold, Stephan Nachhaltigkeitseffekte durch Herstellung und Anwendung nanotechnologischer Produkte. Schriftenreihe des IÖW 177/04 (2004).

20. Warheit, David B. Comparative Pulmonary Toxicity Assessment on Single Walled Carbon Nano Tubes in Rats. In: Toxicol. Sci. 77(1): 117 (2004)

21. Warheit, David B. Nanoparticles: Health impacts? Materials today 02/2004 pp. 32-35 (2004b)

22. Zhiyong Tang, Nicholas A. Kotov, Michael Giersig, Spontaneous Organization of Single CdTe Nanoparticles into Luminescent Nanowires, Science 297( 5579): 237 - 240 (2002). 